\title{
Approximate Method to Evaluate Reliability of Complex Networks
}

\author{
Petru Cașcaval \\ Department of Computer Science and Engineering, "Gheorghe Asachi” Technical University of Iaşi, Dimitrie Mangeron Street, 27, \\ 700050 Iaşi, Romania
}

Correspondence should be addressed to Petru Caşcaval; cascaval@cs.tuiasi.ro

Received 20 April 2018; Revised 12 July 2018; Accepted 31 July 2018; Published 12 November 2018

Academic Editor: Ireneusz Czarnowski

Copyright (c) 2018 Petru Caşcaval. This is an open access article distributed under the Creative Commons Attribution License, which permits unrestricted use, distribution, and reproduction in any medium, provided the original work is properly cited.

This paper deals with the issue of reliability evaluation in complex networks, in which both link and node failures are considered, and proposes an approximate method based on the minimal paths between two specified nodes. The method requires an algorithm for transforming the set of minimal paths into a sum of disjoint products (SDP). To reduce the computation burden, in the first stage, only the links of the network are considered. Then, in the second stage, each term of the set of disjoint link-products is separately processed, taking into consideration the reliability values for both links and adjacent nodes. In this way, a reliability expression with a one-to-one correspondence to the set of disjoint products is obtained. This approximate method provides a very good accuracy and greatly reduces the computation for complex networks.

\section{Introduction}

The network reliability theory is extensively applied in many real-world systems that can be modeled as stochastic networks, such as communication networks, sensor networks, social networks, etc. A variety of tools are used for system modeling and computation of reliability or availability indices that describe in a certain way the ability of a network to carry out a desired operation. Most tools are based on algorithms described in terms of minimal path set or minimal cut set (see, for example, [1-8]). Unfortunately, the problem of computing the network reliability based on the set of the minimal paths or cuts is NP-hard [7, 9]. For this reason, in case of more complex networks, other techniques for approximate reliability evaluation are also applied, such as those based on network decomposition or on Monte Carlo simulations (see, for example, [10-16]).

In this work, we deal with the problem of evaluation of two-terminal reliability or availability indices in mediumto-large networks, based on SDP algorithms, in which both link and node failures are considered.

Many authors address this problem assuming that the nodes of the system are perfectly reliable (see, for example, $[1,4-6])$. However, in a communication system, nodes also have certain probability of failure so that the reliability evaluation assuming perfect nodes is not realistic.

The failure of a node inhibits the work of all links connected to it. Based on this concept, starting from the given network with unreliable nodes, reduced models with perfect nodes but with links having increased failure probabilities can be obtained. This method is simple, but not so accurate. Because the failure of a node inhibits the work of all adjacent links, the work of the links connected to it depends on the state of this common node. However, a reduced model is solved under the hypothesis according to which the failures that affect the network are independent. For this reason, the reliability estimation must be accepted with caution. Indeed, the estimation error of two-terminal network reliability could be unacceptable in many cases, especially when the failure probabilities of the nodes have high values.

To highlight this aspect, let us consider a simple network with unreliable nodes as presented in Figure 1(a). The reliability of the connection between nodes 1 and 4 has to be evaluated. These two terminal nodes are considered in series with the rest of the network. Three reduced models with perfect nodes and links having increased probabilities of failure are presented in Figures 1(b)-1(d). With dashed line, it is indicated that the failure of a node can be modeled by a cut 


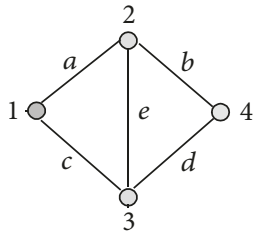

(a) Unreliable nodes

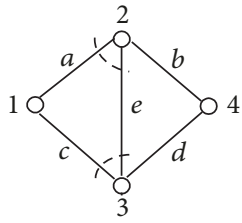

(c) Perfect (b) Perfect

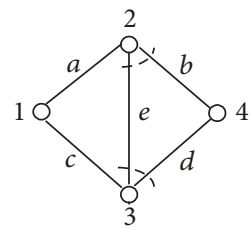

(d) Perfect

nodes-model 3

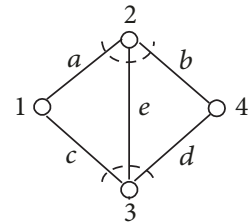

nodes-model 1

Figure 1: A simple network: (a) initial model; (b-d) reduced models.

of the links connected to it. Consequently, in case of a reduced model with perfect nodes, the reliability of the links in the network must be adjusted accordingly. It is easy to observe that other reduced models are also possible.

For the reduced models presented in Figure 1, Table 1 shows how the reliability of each link in the network is adjusted to capture the fact that the nodes of the given network are also unreliable.

For a numerical evaluation of these approximate models, let us consider a network with the following reliability values for the nodes and the links: $p_{1}=0.99, p_{2}=0.98, p_{3}=0.97$, $p_{4}=0.96, p_{a}=0.99, p_{b}=0.98, p_{c}=0.97, p_{d}=0.96$, and $p_{e}=$ 0.95 . The numerical results expressing the reliability estimation of the connection between nodes 1 and $4\left(R_{1-4}\right)$ are presented in Table 2.

These numerical results show that the reduced models with perfect nodes might be useful in a way, but the reliability estimation is not so accurate, even for this simple network.

A better solution that makes a link-based reliability evaluation algorithm adaptable to communication systems is given by Aggarwal et al. [2]. Thus, based on a SDP expression obtained with the assumption of perfect nodes, the node reliability values are taken into account in a specific mode for each term of the set of disjoint link-products. However, the authors do not completely address aspects of the influence of a node on the links connected to it, as it will be seen in Section 5. Moreover, the method is limited to the SDP expressions generated with a so-called "single variable inversion" (SVI) technique. But, for complex networks, "multiple variable inversion" (MVI) techniques are required $[1,4,14,16]$.

In this work, a new approximate method for twoterminal network reliability evaluation with a much better accuracy is proposed. The method is based on algorithms described in terms of minimal path set and covers both SVI and MVI expressions. Just like in [2], in the first stage, the method is focused only on the links of the network. For the any two given nodes, all the minimal paths are enumerated,
TABLE 1: Adjusted reliability values for the links in the network.

\begin{tabular}{|c|c|c|}
\hline $\begin{array}{l}\text { Reduced } \\
\text { model }\end{array}$ & \multicolumn{2}{|c|}{ New reliability values } \\
\hline Model 1 & \multicolumn{2}{|c|}{$p_{a}^{\prime}=p_{a} p_{2}, p_{b}^{\prime}=p_{b} p_{2}, p_{c}^{\prime}=p_{c} p_{3}, p_{d}^{\prime}=p_{d} p_{3}, p_{e}^{\prime}=p_{e} p_{2} p_{3}$} \\
\hline Model 2 & \multicolumn{2}{|c|}{$p_{a}^{\prime}=p_{a} p_{2}, p_{b}^{\prime}=p_{b}, p_{c}^{\prime}=p_{c} p_{3}, p_{d}^{\prime}=p_{d}, p_{e}^{\prime}=p_{e} p_{2} p_{3}$} \\
\hline Model 3 & \multicolumn{2}{|c|}{$p_{a}^{\prime}=p_{a}, p_{b}^{\prime}=p_{b} p_{2}, p_{c}^{\prime}=p_{c}, p_{d}^{\prime}=p_{d} p_{3}, p_{e}^{\prime}=p_{e} p_{2} p_{3}$} \\
\hline \multicolumn{3}{|c|}{ TABLE 2: Numerical results $\left(R_{1-4}\right)$. } \\
\hline \multirow[t]{2}{*}{ Exact result } & \multicolumn{2}{|c|}{$\begin{array}{l}\text { Approximate results obtained } \\
\text { based on the reduced models }\end{array}$} \\
\hline & Model $1 \quad$ Model 2 & Model 3 \\
\hline 0.9467 & 0.9477 & 0.9476 \\
\hline
\end{tabular}

and then this set of minimal paths is transformed into a set of disjoint products. In the second stage, each term of the sum of disjoint products including state variables associated to the links is processed distinctly by considering both links and adjacent node reliability values.

This new approximate method reduces the computation time for large networks to a great extent, compared with an exact method. This reduction in computation time is explained by the fact that the node failures are taken into account only in the second stage when the computation process is simpler, belonging to the $O(n \times m)$ class of complexity, where $n$ is the number of disjoint link-products and $m$ is the number of the network components.

The rest of this paper is organized as follows. Section 2 introduces notations, assumptions, and a short nomenclature, while Section 3 presents general issues regarding the problem of network reliability evaluation. Section 4 provides a method for exact evaluation of two-terminal network reliability when both node and link failures are considered. Section 5, the most extensive one, presents a new approximate method that reduces the complexity of this problem in medium-to-large networks. Section 6 presents some obtained numerical results. The paper ends with some final remarks presented in Section 7.

\section{Notations and Preliminary Considerations}

\subsection{Nomenclature}

(a) Reliability: the two-terminal reliability of a stochastic network expresses the probability that there exists at least one path between any two specified nodes (let us say a source node and a target one) which operate successfully

(b) Connected nodes: two nodes which can communicate with each other are connected; otherwise, they are disconnected

(c) Minimal path: a minimal set of links and their adjacent nodes whose good operation ensures that two given nodes are connected. For a minimal path, any proper subset is no longer a path 
(d) Uniproduct: Boolean product composed only of distinct uncomplemented variables

(e) Subproduct: part of a Boolean product that is a complemented or an uncomplemented uniproduct

(f) Mixproduct: product of one uncomplemented subproduct and one or more complemented subproducts

(g) Disjoint products: a set of products expressing mutually exclusive states

\subsection{Notations}

(a) $G(V, E)$ is a network model with node set $V=\left\{y_{1}\right.$, $\left.y_{2}, \ldots, y_{k}\right\}$ and link set $E=\left\{x_{1}, x_{2}, \ldots, x_{m}\right\}$

(b) $s, t \in V, s \neq t$, are the source and target nodes

(c) $p_{x}$ is the reliability of node $x \in V$ or link $x \in E$, and $q_{x}=1-p_{x}$

(d) $R_{s-t}$ is the two-terminal reliability of network $G(V, E)$ with $s$ and $t$ the source and target nodes $(s-t$ network reliability)

(e) $P(A)$ denotes the probability of the event $A$

\subsection{Assumptions}

(a) Each component in the network (i.e., node or link) is either operational or failed, so a logical variable is used to indicate its state. The same notations $y_{1}, y_{2}, \ldots, y_{k}$ and $x_{1}, x_{2}, \ldots, x_{m}$ are used to denote these logical variables

(b) The events of failure that affect the nodes or the links in network are stochastically independent

\section{Considerations on Network Reliability Evaluation}

Consider $G(V, E)$ the network under study and $s, t \in V, s \neq t$, the source and target nodes. For this model, consider the minimal path set MPS $=\left\{P_{1}, P_{2}, \ldots, P_{n p}\right\}$. Note that a minimal path $P_{i} \in$ MPS is expressed by a product of distinct logical variables associated with some links or nodes of the network, and the reliability of this path is given by

$$
P\left(P_{i}\right)=\prod_{c \in P_{i}} p_{c}
$$

Starting from this minimal path set, a structure function $S=\bigcup_{i=1}^{n p} P_{i}$ is defined, and the two-terminal network reliability of this model is calculated by

$$
R_{s-t}=P(S)=P\left(\bigcup_{i=1}^{n p} P_{i}\right) .
$$

Efficient methods for enumerating all minimal paths are presented in $[14,17,18]$. To compute the network reliability
$R_{s-t}$ based on (2), the well-known rule of sum of disjoint products is recommended:

$$
\begin{aligned}
P\left(\bigcup_{i=1}^{n} A_{i}\right)= & P\left(A_{1}\right)+P\left(\bar{A}_{1} \cap A_{2}\right)+P\left(\bar{A}_{1} \cap \bar{A}_{2} \cap A_{3}\right) \\
& +\cdots+P\left(\bar{A}_{1} \cap \bar{A}_{2} \cap \cdots \cap \bar{A}_{n-1} \cap A_{n}\right) .
\end{aligned}
$$

For this purpose, the structure function $S$ is transformed into an equivalent form $S^{\prime}$, composed only of disjoint products (DP), so that the two-terminal network reliability $R_{s-t}$ is given by

$$
R_{s-t}=P\left(S^{\prime}\right)=\bigcup_{j} \mathrm{DP}_{j}=\sum_{j} P\left(\mathrm{DP}_{j}\right)
$$

Observe that (4) is easy to compute, so that the problem of computing the two-terminal network reliability essentially boils down to generating a new set of disjoint products starting from the set MPS of minimal paths. Unfortunately, this task falls in the NP-hard category.

The first computerized SDP algorithm was proposed by Aggarwal et al. [3], but one of the best known SDP algorithms for transforming the structure function to a sum of disjoint products is given by Abraham [4].

If $P$ and $Q$ are two undisjoint products, and $x_{1}, x_{2}, \ldots$, $x_{s} \in P \backslash Q$, according to Abraham's theorem, the following logical expression can be written as follows:

$$
P+Q=P+\bar{x}_{1} Q+x_{1} \bar{x}_{2} Q+x_{1} x_{2} \bar{x}_{3} Q+\cdots+x_{1} x_{2} \cdots x_{s-1} \bar{x}_{s} Q .
$$

Note that, to ensure that two products are disjoint, only a single complemented variable is added with each new term. Abraham's algorithm is a reference for the socalled SVI algorithms. Two improved SVI algorithms are presented in $[19,20]$.

To reduce the computation time, other approaches based on the so-called MVI technique have been devised (see, for example, $[5,6,21-23])$.

When an MVI technique is applied, a product may contain distinct logical variables (complemented or not) but also one or more complemented subproducts. For instance, take seven variables representing a network state where links 2 and 4 are not both operational, link 6 is operational, link 7 is in the failed state, and links 1,3 , and 5 are in a do-notcare state. In an MVI approach, this network state is represented by the Boolean expression $\overline{x_{2} x_{4}} x_{6} \bar{x}_{7}$, whereas in an SVI approach, by the expression $\overline{x_{2}} x_{6} \overline{x_{7}}+x_{2} \overline{x_{4}} x_{6} \overline{x_{7}}$, so that the advantage of the MVI approach is obvious.

An excellent survey on MVI techniques can be found in [16]. A new MVI technique, called NMVI, is proposed by Caşcaval and Floria in [1].

According to the NMVI method, in order to expand a product $Q$ in relation to a given uniproduct $P$, so that any new generated product to be disjoint with $P$, the following two MVI rules are applied. 
Rule 1. Type I expansion

If $x_{1}, x_{2}, \ldots, x_{s} \in P \backslash Q$, the following equation can be written as follows:

$$
P+Q=P+x_{1} x_{2} \cdots x_{s} Q+\bar{x}_{1} x_{2} \cdots x_{s} Q
$$

When $P$ and $Q$ are both uniproducts, for the new term $x_{1} x_{2}$ $\cdots x_{s} Q$, the absorption law is applicable, so that a reduced logical expression with two disjoint products is obtained:

$$
P+Q=P+{\overline{x_{1} x_{2} \cdots x_{s}}} \text {. }
$$

Rule 2. Type II expansion

Consider $P=x_{1} x_{2} \cdots x_{i} R_{1}$, and $Q=\overline{x_{1} x_{2} \cdots x_{i} x_{i+1} \cdots x_{s}} R_{2}$. By applying the Boolean rule $\overline{x y}=\bar{x}+x \bar{y}$, the following logical expression results are as follows:

$$
\begin{aligned}
P+Q & =P+\overline{x_{1} x_{2} \cdots x_{i} x_{i+1} \cdots x_{s}} R_{2} \\
& =P+\overline{x_{1} x_{2} \cdots x_{i}} R_{2}+x_{1} x_{2} \cdots x_{i} \overline{x_{i+1} \cdots x_{s}} R_{2} .
\end{aligned}
$$

When $R_{1} \in R_{2}$, the term $x_{1} x_{2} \ldots x_{i} \overline{x_{i+1}} \cdots x_{s} R_{2}$ is absorbed by product $P$, so that a reduced logical expression composed of two disjoint products is obtained:

$$
P+Q=P+\overline{x_{1} x_{2} \cdots x_{i}} R_{2} .
$$

As shown in [1], NMVI is an efficient method, providing fewer disjoint products compared with other well-known MVI methods, as CAREL [5], VT [6], or KDH88 [21].

In the next two sections, we address the problem of twoterminal network reliability evaluation, in which both link and node failures are considered. First, an exact method of reliability evaluation is discussed. Then, a new approximate method is presented, with the advantage of being much faster and able to offer a very good accuracy.

\section{Exact Evaluation of Network Reliability}

For two given nodes, $s$ and $t$, an exact evaluation of twoterminal network reliability can be obtained based on the set of minimal paths that include both links and adjacent nodes. Compared with the case in which the study is limited to the links of the network, when the nodes are also considered, the number of the minimal paths is unchanged, but any term is extended by also including the adjacent nodes. To illustrate this method, let us analyze the network $N_{1}$ presented in Figure 2(a), where the source and target nodes are 1 and 5. These two terminal nodes are considered in series with the rest of the network. For these two given nodes, the set of minimal paths is

$$
\begin{aligned}
\mathrm{MPS}= & \{3 b f, 34 \mathrm{beg}, 24 a d g, 23 a c f, \\
& 234 a c e g, 234 a d e f, 234 b c d g\} .
\end{aligned}
$$

By applying the NMVI method, the following set of disjoint products results as follows:

$$
\begin{aligned}
\mathrm{DPS}=\{ & 3 b f, 34 b e g \bar{f}, 24 a d f g \overline{3 b}, 24 a d g \bar{f} \overline{3 b e}, 23 a c f \bar{b} \overline{4 d g}, \\
& 234 a c e g \bar{b} \bar{d} \bar{f}, 234 a d e f \bar{b} \bar{c} \bar{g}, 234 b c d g \bar{a} \bar{e} \bar{f}\}
\end{aligned}
$$

Finally, the reliability $R_{1-5}$ is given by

$$
\begin{aligned}
R_{1-5}= & p_{1} p_{5}\left(p_{3} p_{b} p_{f}+p_{3} p_{4} p_{b} p_{e} p_{g}\left(1-p_{f}\right)\right. \\
& +p_{2} p_{4} p_{a} p_{d} p_{g}\left(p_{f}\left(1-p_{3} p_{b}\right)\right. \\
& \left.+\left(1-p_{f}\right)\left(1-p_{3} p_{b} p_{e}\right)\right) \\
& +p_{2} p_{3} p_{a} p_{c} p_{f}\left(1-p_{b}\right)\left(1-p_{4} p_{d} p_{g}\right) \\
& +p_{2} p_{3} p_{4}\left(p_{a} p_{c} p_{e} p_{g}\left(1-p_{b}\right)\left(1-p_{d}\right)\left(1-p_{f}\right)\right. \\
& +p_{a} p_{d} p_{e} p_{f}\left(1-p_{b}\right)\left(1-p_{c}\right)\left(1-p_{g}\right) \\
& \left.\left.+p_{b} p_{c} p_{d} p_{g}\left(1-p_{a}\right)\left(1-p_{e}\right)\left(1-p_{f}\right)\right)\right)
\end{aligned}
$$

The same network is analyzed in [2], example 2. So, we compared the numerical result obtained based on this equation with the result generated with equation (21) presented in [2]. These results are identical. For example, assuming for all the nodes a reliability of 0.98 and for all the links a reliability of 0.95 , both methods give a reliability value $R_{1-5}=0.969611$.

To cover both nodes and links, much more logical variables are used. The problem that arises in this case is that the number of disjoint products increases to a large extent when complex networks are evaluated. To highlight this aspect, comparative results with respect to the network models $N_{2}$ and $N_{3}$ given in Figure 2 are presented in Table 3.

Compared with the case in which the study is limited to the links of the network, when the adjacent nodes are also considered, the number of disjoint products increases significantly. The relative growth with respect to the number of disjoint products is about $39 \%$ for network $N_{2}$, but for the more complex network $N_{3}$, this relative growth reaches $86 \%$.

\section{Approximate Approach for Network Reliability Evaluation}

The process of generating the set of disjoint products is a difficult one, of NP-hard complexity. In order to reduce the computation time, the process of enumerating the minimal paths and their development as a sum of disjoint products is focused only to the links of the network. For this purpose, for a link $x_{i} \in E$, let $X_{i}$ be a logical variable that reflects the event of successful communication through that branch-that means that the link $x_{i}$ and the two adjacent nodes are operational. Thus, the structure function $S$ can be expressed in terms of these logical variables $X_{1}, X_{2}, \ldots, X_{m}$. 


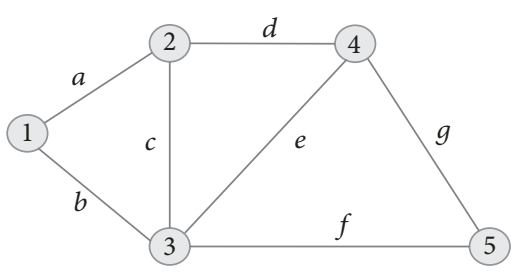

(a) 5-node, 7-link network $\left(N_{1}\right)$

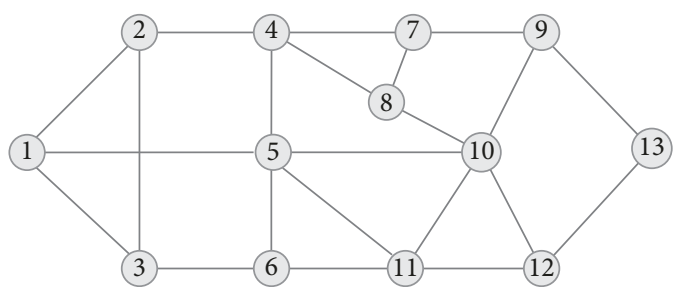

(b) 13-node, 22-link network $\left(N_{2}\right)$

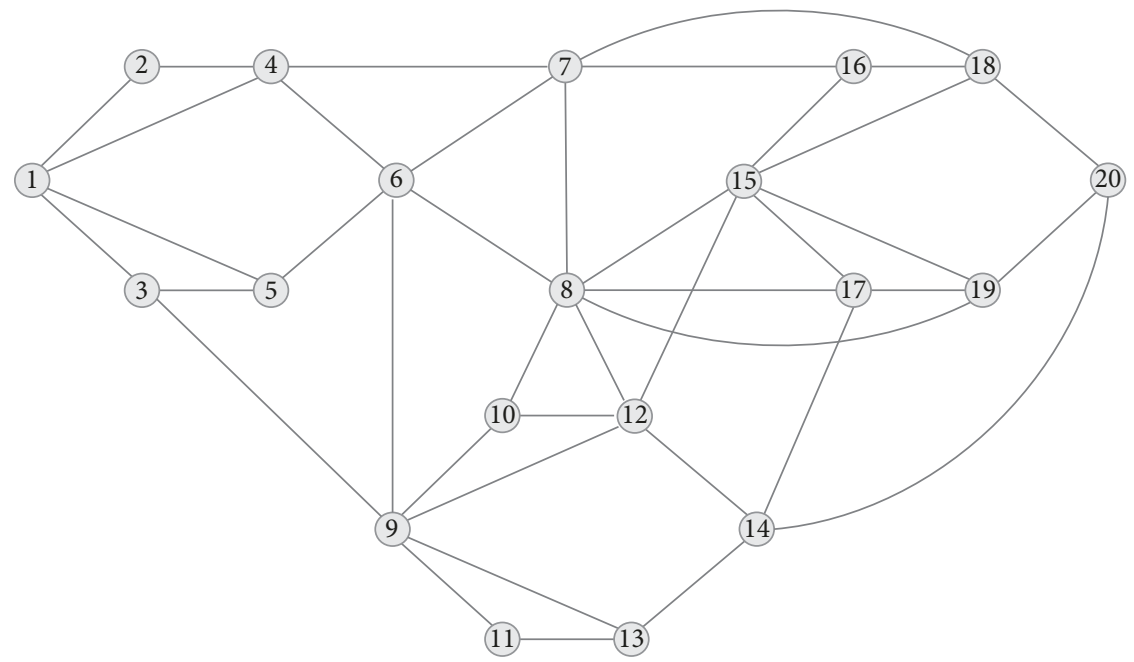

(c) 20-node, 40-link network $\left(N_{3}\right)$

Figure 2: Network models with unreliable nodes: (a) 5-node, 7-link network $\left(N_{1}\right)$; (b) 13-node, 22-link network $\left(N_{2}\right)$; (c) 20-node, 40-link network $\left(N_{3}\right)$.

TABLE 3: The number of disjoint products. Comparative results.

\begin{tabular}{|c|c|c|c|}
\hline \multirow{2}{*}{$\begin{array}{l}\text { Network } \\
\text { models }\end{array}$} & \multirow{2}{*}{$\begin{array}{c}\text { Number of } \\
\text { minimal } \\
\text { paths }\end{array}$} & \multicolumn{2}{|c|}{$\begin{array}{l}\text { Number of disjoint products } \\
\text { generated by NMVI, including }\end{array}$} \\
\hline & & Only links & Both links and nodes \\
\hline $\mathrm{N}_{2}$ & 281 & 2269 & 3151 \\
\hline$N_{3}$ & 16618 & 1799888 & 3353457 \\
\hline
\end{tabular}

In the second stage, each term of the sum of disjoint products is processed distinctly by considering both links and adjacent node reliability values. The node reliability values are taken into account in a specific mode for each term of the set of disjoint link-products, when only the adjacent nodes of the links that compose the current product are considered. This is the starting point for this approximate approach.

Based on the set of disjoint products DPS $=\left\{\mathrm{DP}_{1}, \mathrm{DP}_{2}\right.$, $\left.\ldots, \mathrm{DP}_{n}\right\}$, the two-terminal network reliability is computed by applying (4).

A term DP in the set of disjoint products is a mixproduct that includes one uniproduct, noted with $U$, and one or more complemented subproducts. Figure 3 shows such a complex mixproduct.

As illustrated in Figure 3, the uniproduct $U$ reflects a state of operability of a part of the network that ensures the connection between the source and target nodes. Let SAN be the set of all adjacent nodes of the links that compose the uniproduct $U$. All these links and all the nodes that belong to SAN are operational. Consequently, the probability of the network state described by $U$ is given by

$$
P(U)=\prod_{x \in U} p_{x} \prod_{y \in \text { SAN }} p_{y}
$$

The main problem is how to compute or at least evaluate with a good accuracy the probability of a network state described by a complemented subproduct (such a subproduct is illustrated in Figure 3 with a dashed line).

A complemented subproduct reflects a state of inoperability of a branch or of a bigger portion of the network. To begin with, consider the case where such a portion of the network is independent of that portions described by the other complemented subproducts. Under these circumstances, the current term can be independently evaluated. Two cases are distinguished.

Case 1 (a single complemented variable (an SVI term)). Consider a single complemented variable $\overline{X_{i}}$ (an SVI term) associated with the link $x_{i}$ that connects two nodes; let us say $y_{i}$ and $y_{j}$ (for instance, the variable $\overline{X_{11}}$ in Figure 3 ). The probability of this event is

$$
P\left(\overline{X_{i}}\right)=1-p_{x_{i}}^{\prime}
$$




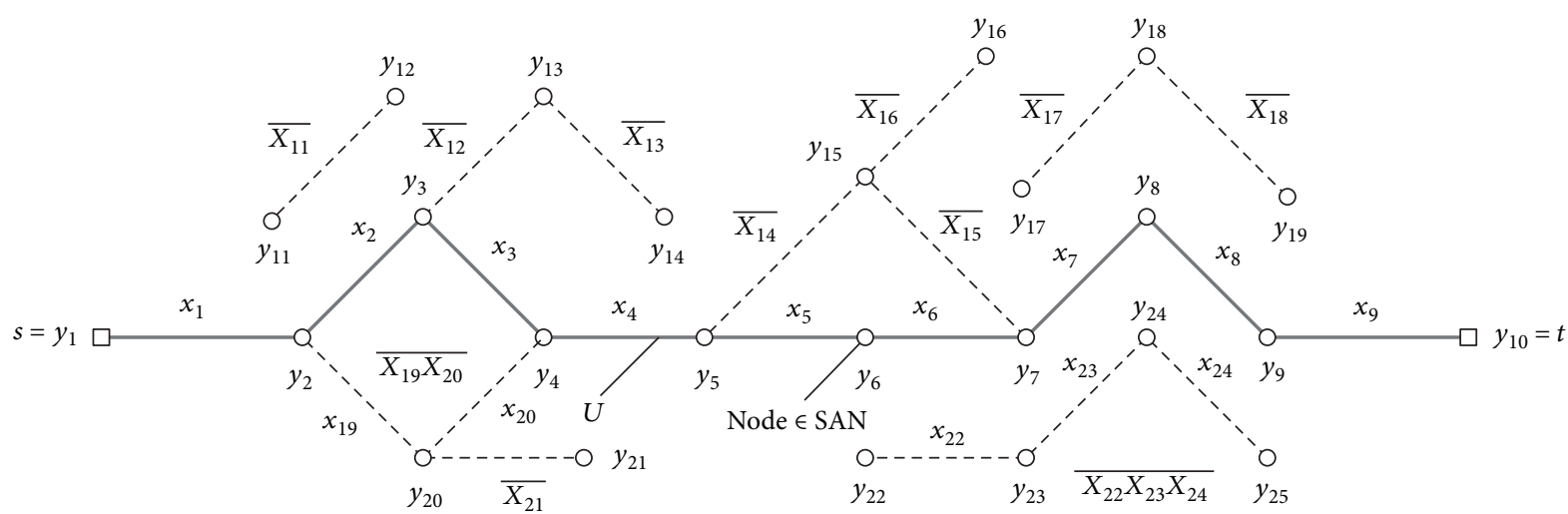

Figure 3: Illustration of a complex mixproduct: $\mathrm{DP}=X_{1} X_{2} \cdots X_{9} \overline{X_{11}} \overline{X_{12}} \cdots \overline{X_{18}} \overline{X_{19} X_{20}} \overline{X_{21}} \overline{X_{22} X_{23} X_{24}}$.

where

$$
p_{x_{i}}^{\prime}= \begin{cases}p_{x_{i}} p_{y_{i}}, & \text { if } y_{j} \in \mathrm{SAN}, y_{i} \notin \mathrm{SAN}, \\ p_{x_{i}} p_{y_{j}}, & \text { if } y_{i} \in \mathrm{SAN}, y_{j} \notin \mathrm{SAN}, \\ p_{x_{i}} p_{y_{i}} p_{y_{j}}, & \text { if } y_{i}, y_{j} \notin \mathrm{SAN} .\end{cases}
$$

Equations (13) and (14) are found in another form in [2] where the same problem of network reliability evaluation is treated. Remember that the method presented in [2] covers only SDP expressions composed of SVI terms.

Case 2 (an MVI term). Consider a complemented subproduct $\overline{X_{1} X_{2} \cdots X_{k}}$ (an MVI term) that describes a state of inoperability of a portion of the network as illustrated in Figure 4. The probability that this portion of the network to be inoperable is

$$
P\left(\overline{X_{1} X_{2} \cdots X_{k}}\right)=1-Q
$$

where the product $Q$ includes not only the reliability of the corresponding links but also the reliability of the adjacent nodes that do not belong to SAN, considered only once. More exactly, the probability $Q$ is computed by the following sequence of steps presented in Pseudocode 1.

Even though the two portions of the network described by two complemented subproducts may not have any common link, they may have one or even more common nodes. Consequently, the state of inoperability of these two portions of the network may be due to the failure of such a common node. Of course, we refer to a common node that does not belong to SAN.

In the first stage, the analysis of these dependencies given by the common nodes is limited to the level of pairs of complemented subproducts. The following three cases are distinguished.

Case 3 (two SVI terms with a common node). Consider two complemented variables $\overline{X_{i}}$ and $\overline{X_{j}}$ that describe a state of inoperability for two branches $x_{i}$ and $x_{j}$ that have

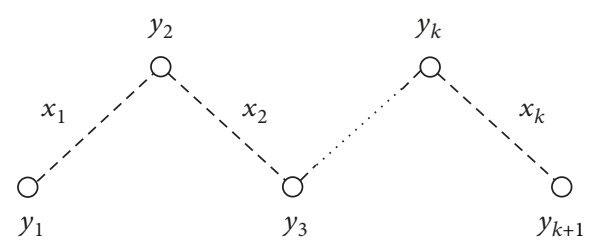

Figure 4: Illustration of a MVI term: $\overline{X_{1} X_{2} \cdots X_{k}}$.

$$
\begin{aligned}
& \begin{array}{l}
Q=1 \text {; } \\
\text { for } i=1: k
\end{array} \\
& \qquad Q=Q^{*} p_{x_{i}} \text {; } \\
& \quad \text { if } y_{i} \notin \text { SAN then } Q=Q^{*} p_{y_{i}} \text {; } \\
& \text { end } \\
& \text { if } y_{k+1} \notin \text { SAN then } Q=Q^{*} p_{y_{k+1}} \text {; }
\end{aligned}
$$

Pseudocode 1: Computing the product $Q$.

$y_{k}$ as a common node, as illustrated in Figure 5. The node $y_{k} \notin \mathrm{SAN}$.

Let us define the probabilities $p_{x_{i}}^{\prime}$ and $p_{x_{j}}^{\prime}$ associated with the links $x_{i}$ and $x_{j}$, given as follows:

$$
\begin{aligned}
& p_{x_{i}}^{\prime}= \begin{cases}p_{x_{i}}, & \text { if } y_{i} \in \mathrm{SAN}, \\
p_{x_{i}} p_{y_{i}}, & \text { if } y_{i} \notin \mathrm{SAN},\end{cases} \\
& p_{x_{j}}^{\prime}= \begin{cases}p_{x_{j}}, & \text { if } y_{j} \in \mathrm{SAN}, \\
p_{x_{j}} p_{y_{j}}, & \text { if } y_{j} \notin \mathrm{SAN} .\end{cases}
\end{aligned}
$$

By applying the theorem of total probability to the event space $\left\{y_{k}, \bar{y}_{k}\right\}$, the following equation can be written as follows:

$$
P\left(\overline{X_{i}} \overline{X_{j}}\right)=P(X)=p_{y_{k}} P\left(X \mid y_{k}\right)+\left(1-p_{y_{k}}\right) P\left(X \mid \bar{y}_{k}\right) .
$$




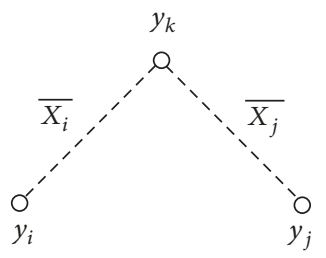

FIGURE 5: Illustration of two inoperable links with a common node.

As

$$
\begin{aligned}
& P\left(X \mid \bar{y}_{k}\right)=1, \\
& P\left(X \mid y_{k}\right)=\left(1-p_{x_{i}}^{\prime}\right)\left(1-p_{x_{j}}^{\prime}\right),
\end{aligned}
$$

finally, the equation becomes

$$
P\left(\overline{X_{i}} \overline{X_{j}}\right)=1-p_{y_{k}}\left(p_{x_{i}}^{\prime}+p_{x_{j}}^{\prime}-p_{x_{i}}^{\prime} p_{x_{j}}^{\prime}\right)
$$

Note that this case is not treated in [2].

Case 4 (an MVI term and an SVI one with a common node). Consider, for example, $\overline{X_{1} X_{2} X_{3}}$ and $\overline{X_{4}}$ to be two terms in DP describing a state of inoperability of two portions of the network as illustrated in Figure 6 . The common node $y_{2} \notin \mathrm{SAN}$.

Let us define the probabilities $p_{x_{1}}^{\prime}, p_{x_{2}}^{\prime}, p_{x_{3}}^{\prime}$, and $p_{x_{4}}^{\prime}$ given as follows:

$$
\begin{aligned}
& p_{x_{1}}^{\prime}= \begin{cases}p_{x_{1}}, & \text { if } y_{1} \in \mathrm{SAN}, \\
p_{x_{1}} p_{y_{1}}, & \text { if } y_{1} \notin \mathrm{SAN},\end{cases} \\
& p_{x_{2}}^{\prime}= \begin{cases}p_{x_{2}}, & \text { if } y_{3} \in \mathrm{SAN}, \\
p_{x_{2}} p_{y_{3}}, & \text { if } y_{3} \notin \mathrm{SAN},\end{cases} \\
& p_{x_{3}}^{\prime}= \begin{cases}p_{x_{3}}, & \text { if } y_{4} \in \mathrm{SAN}, \\
p_{x_{3}} p_{y_{4}}, & \text { if } y_{4} \notin \mathrm{SAN},\end{cases} \\
& p_{x_{4}}^{\prime}= \begin{cases}p_{x_{4}}, & \text { if } y_{5} \in \mathrm{SAN}, \\
p_{x_{4}} p_{y_{5}}, & \text { if } y_{5} \notin \mathrm{SAN},\end{cases}
\end{aligned}
$$

By applying the theorem of total probability to the event space $\left\{y_{2}, \bar{y}_{2}\right\}$, the following equation can be written as follows:

$P\left(\overline{X_{1} X_{2} X_{3}} \overline{X_{4}}\right)=P(X)=p_{y_{2}} P\left(X \mid y_{2}\right)+\left(1-p_{y_{2}}\right) P\left(X \mid \bar{y}_{2}\right)$.

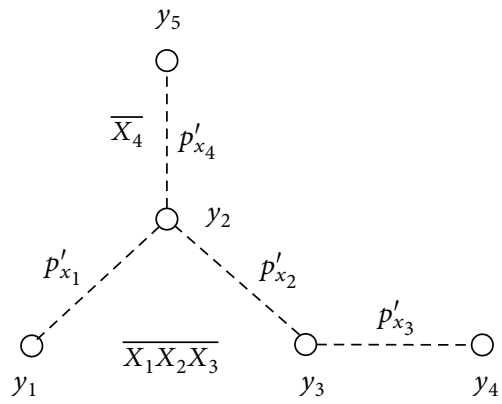

Figure 6: Illustration of an MVI term and an SVI one with a common node.

As

$$
\begin{aligned}
& P\left(X \mid \bar{y}_{2}\right)=1, \\
& P\left(X \mid y_{2}\right)=\left(1-p_{x_{1}}^{\prime} p_{x_{2}}^{\prime} p_{x_{3}}^{\prime}\right)\left(1-p_{x_{4}}^{\prime}\right),
\end{aligned}
$$

finally, the following equation results in

$$
P\left(\overline{X_{1} X_{2} X_{3}} \overline{X_{4}}\right)=1-p_{y_{2}}\left(p_{x_{1}}^{\prime} p_{x_{2}}^{\prime} p_{x_{3}}^{\prime}+p_{x_{4}}^{\prime}-p_{x_{1}}^{\prime} p_{x_{2}}^{\prime} p_{x_{3}}^{\prime} p_{x_{4}}^{\prime}\right) .
$$

Note that, if the two events were treated independently, the following equation would result in

$$
\begin{aligned}
P\left(\overline{X_{1} X_{2} X_{3}}\right) P\left(\overline{X_{4}}\right) & =\left(1-p_{x_{1}}^{\prime} p_{x_{2}}^{\prime} p_{x_{3}}^{\prime} p_{y_{2}}\right)\left(1-p_{x_{4}}^{\prime} p_{y_{2}}\right) \\
& =1-p_{y_{2}}\left(p_{x_{1}}^{\prime} p_{x_{2}}^{\prime} p_{x_{3}}^{\prime}+p_{x_{4}}^{\prime}-p_{x_{1}}^{\prime} p_{x_{2}}^{\prime} p_{x_{3}}^{\prime} p_{x_{4}}^{\prime} p_{y_{2}}\right) \\
& <P\left(\overline{X_{1} X_{2} X_{3} X_{4}}\right) .
\end{aligned}
$$

Remark 1. Equation (25) shows that when a common node is not taken into account, the reliability estimation is a pessimistic one.

Case 5 (two MVI terms with a common node). Consider, for example, $\overline{X_{1} X_{2}}$ and $\overline{X_{3} X_{4}}$ to be the two MVI terms describing a state of inoperability of two parts of the network, as illustrated in Figure 7, where the communication between nodes 1 and 2 and between nodes 3 and 4 is not possible. The common node $y_{5} \notin$ SAN.

Let us define the probabilities $p_{x_{1}}^{\prime}, p_{x_{2}}^{\prime}, p_{x_{3}}^{\prime}$, and $p_{x_{4}}^{\prime}$ given by the following:

$$
p_{x_{i}}^{\prime}= \begin{cases}p_{x_{i}}, & \text { if } y_{i} \in \mathrm{SAN}, \\ p_{x_{i}} p_{y_{i}}, & \text { if } y_{i} \notin \mathrm{SAN}, \\ i=1,2,3,4 . & \end{cases}
$$




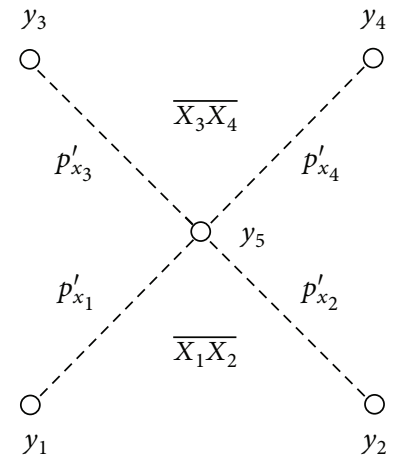

FIGURe 7: Illustration of two MVI terms with a common node.

The probability of this state, $P\left(\overline{X_{1} X_{2}} \overline{X_{3} X_{4}}\right)$, can be determined by applying the rule of total probability to the event space $\left\{y_{5}, \bar{y}_{5}\right\}$.

If $X=\overline{X_{1} X_{2}} \overline{X_{3} X_{4}}$, the following equation can be written as follows:

$$
P(X)=p_{y_{5}} P\left(X \mid y_{5}\right)+\left(1-p_{y_{5}}\right) P\left(X \mid \bar{y}_{5}\right) .
$$

Obviously, $P\left(X / \bar{y}_{5}\right)=1$.

$$
\begin{aligned}
P\left(X \mid y_{5}\right) & =\left(1-p_{x_{1}}^{\prime} p_{x_{2}}^{\prime}\right)\left(1-p_{x_{3}}^{\prime} p_{x_{4}}^{\prime}\right) \\
& =1-p_{x_{1}}^{\prime} p_{x_{2}}^{\prime}-p_{x_{3}}^{\prime} p_{x_{4}}^{\prime}+p_{x_{1}}^{\prime} p_{x_{2}}^{\prime} p_{x_{3}}^{\prime} p_{x_{4}}^{\prime} .
\end{aligned}
$$

Finally, the equation becomes

$$
P\left(\overline{X_{1} X_{2}} \overline{X_{3} X_{4}}\right)=1-p_{y_{5}}\left(p_{x_{1}}^{\prime} p_{x_{2}}^{\prime}+p_{x_{3}}^{\prime} p_{x_{4}}^{\prime}-p_{x_{1}}^{\prime} p_{x_{2}}^{\prime} p_{x_{3}}^{\prime} p_{x_{4}}^{\prime}\right) .
$$

To exemplify these 5 rules previously defined, consider the mixproduct

$$
\mathrm{DP}=X_{1} X_{2} \cdots X_{9} \overline{X_{11}} \overline{X_{12}} \cdots \overline{X_{18}} \overline{X_{19} X_{20}} \overline{X_{21}} \overline{X_{22} X_{23} X_{24}},
$$

as illustrated in Figure 3. The mixproduct DP includes the uniproduct $U=X_{1} X_{2} \cdots X_{9}$ and for this operable path, the set of adjacent nodes is $\mathrm{SAN}=\left\{y_{1}, y_{2}, \ldots, y_{10}\right\}$.

Taking into account the common nodes for the SVI and MVI terms, the probability of the mixproduct DP can be evaluated with a good accuracy by the following:

$$
\begin{aligned}
P(\mathrm{DP})= & P(U) P\left(\overline{X_{11}}\right) P\left(\overline{X_{12}} \overline{X_{13}}\right) P\left(\overline{X_{14}} \overline{X_{15}}\right) P\left(\overline{X_{16}}\right) \\
& \times P\left(\overline{X_{17}} \overline{X_{18}}\right) P\left(\overline{X_{19} X_{20}} \overline{X_{21}}\right) P\left(\overline{X_{22} X_{23} X_{24}}\right) .
\end{aligned}
$$

By applying the rules presented before, the following equations result in

$$
\begin{aligned}
P(U) & =p_{x_{1}} p_{x_{2}} \cdots p_{x_{9}} p_{y_{1}} p_{y_{2}} \cdots p_{y_{10}}, \\
P\left(\overline{X_{11}}\right) & =1-p_{y_{11}} p_{x_{11}} p_{y_{12}}{ }^{\prime} \\
P\left(\overline{X_{12}} \overline{X_{13}}\right) & =1-p_{y_{13}}\left(p_{x_{12}}+p_{x_{13}}{ }^{\prime}-p_{x_{12}} p_{x_{13}}^{\prime}\right),
\end{aligned}
$$

where $p_{x_{13}}^{\prime}=p_{x_{13}} p_{y_{14}}$.

$$
\begin{aligned}
P\left(\overline{X_{14}} \overline{X_{15}}\right) & =1-p_{y_{15}}\left(p_{x_{14}}+p_{x_{15}}-p_{x_{14}} p_{x_{15}}\right), \\
P\left(\overline{X_{16}}\right) & =1-p_{y_{15}} p_{x_{16}} p_{y_{16}}(\text { an approximate evaluation }), \\
P\left(\overline{X_{17}} \overline{X_{18}}\right) & =1-p_{y_{18}}\left(p_{x_{17}}^{\prime}+p_{x_{18}}^{\prime}-p_{x_{17}}^{\prime} p_{x_{18}}^{\prime}\right),
\end{aligned}
$$

where $p_{x_{17}}^{\prime}=p_{x_{17}} p_{y_{17}}$ and $p_{x_{18}}^{\prime}=p_{x_{18}} p_{y_{19}}$.

$P\left(\overline{X_{19} X_{20}} \overline{X_{21}}\right)=1-p_{y_{20}}\left(p_{x_{19}} p_{x_{20}}+p_{x_{21}}^{\prime}-p_{x_{19}} p_{x_{20}} p_{x_{21}}^{\prime}\right)$,

where $p_{x_{21}}^{\prime}=p_{x_{21}} p_{y_{21}}$.

$$
P\left(\overline{X_{22} X_{23} X_{24}}\right)=1-p_{y_{22}} p_{x_{22}} p_{y_{23}} p_{x_{23}} p_{y_{24}} p_{x_{24}} p_{y_{25}}
$$

Observe that, related to the probability of this mixproduct, an approximation is made with respect to the terms $\overline{X_{14}}, \overline{X_{15}}$, and $\overline{X_{16}}$, because the links $x_{14}, x_{15}$, and $x_{16}$ have a common node, $y_{15} \notin \mathrm{SAN}$. This case is discussed in more detail below.

Case 6 (many terms with a common node). Consider three links $x_{1}, x_{2}$, and $x_{3}$ with a common node and the network state reflected by the SVI terms $\overline{X_{1}}, \overline{X_{2}}$, and $\overline{X_{3}}$, as illustrated in Figure 8.

Suppose that $y_{4} \notin \mathrm{SAN}$. Let us first define the probabilities $p_{x_{1}}^{\prime}, p_{x_{2}}^{\prime}$, and $p_{x_{3}}^{\prime}$ by the following:

$$
p_{x_{i}}^{\prime}= \begin{cases}p_{x_{i}}, & \text { if } y_{i} \in \mathrm{SAN}, \\ p_{x_{i}} p_{y_{i}}, & \text { if } y_{i} \notin \mathrm{SAN}, \\ i=1,2,3 . & \end{cases}
$$

In order to evaluate the probability $P\left(\overline{X_{1}} \overline{X_{2}} \overline{X_{3}}\right)$, the theorem of total probability is applied to the event space $\left\{y_{4}, \bar{y}_{4}\right\}$. The following equation results in

$$
P\left(\overline{X_{1}} \overline{X_{2}} \overline{X_{3}}\right)=P(X)=p_{y_{4}} P\left(X \mid y_{4}\right)+\left(1-p_{y_{4}}\right) P\left(X \mid \bar{y}_{4}\right) .
$$




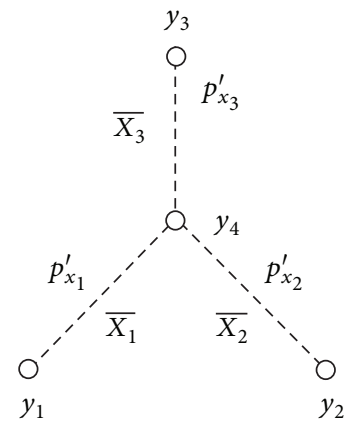

FIGURE 8: Illustration of three SVI terms with a common node.

Obviously, $P\left(X / \bar{y}_{4}\right)=1$. When the node $y_{4}$ is operational, $\overline{X_{1}}, \overline{X_{2}}$, and $\overline{X_{3}}$ reflect independent events, so that

$$
P\left(\overline{X_{1}} \overline{X_{2}} \overline{X_{3}}\right)=P\left(\overline{X_{1}}\right) P\left(\overline{X_{2}}\right) P\left(\overline{X_{3}}\right) .
$$

Consequently, we have

$$
\begin{aligned}
P\left(X \mid y_{4}\right)= & \left(1-p_{x_{1}}^{\prime}\right)\left(1-p_{x_{2}}^{\prime}\right)\left(1-p_{x_{3}}^{\prime}\right) \\
= & 1-p_{x_{1}}^{\prime}-p_{x_{2}}^{\prime}-p_{x_{3}}^{\prime}+p_{x_{1}}^{\prime} p_{x_{2}}^{\prime}+p_{x_{1}}^{\prime} p_{x_{3}}^{\prime} \\
& +p_{x_{2}}^{\prime} p_{x_{3}}^{\prime}-p_{x_{1}}^{\prime} p_{x_{2}}^{\prime} p_{x_{3}}^{\prime} .
\end{aligned}
$$

Finally, the equation becomes

$$
\begin{aligned}
P\left(\overline{X_{1}} \overline{X_{2}} \overline{X_{3}}\right)= & 1-p_{y_{4}}\left(p_{x_{1}}^{\prime}+p_{x_{2}}^{\prime}+p_{x_{3}}^{\prime}-p_{x_{1}}^{\prime} p_{x_{2}}^{\prime}\right. \\
& \left.-p_{x_{1}}^{\prime} p_{x_{3}}^{\prime}-p_{x_{2}}^{\prime} p_{x_{3}}^{\prime}+p_{x_{1}}^{\prime} p_{x_{2}}^{\prime} p_{x_{3}}^{\prime}\right) .
\end{aligned}
$$

Observe that, in case of an exact evaluation, the equation is composed of $2^{3}=8$ terms. This result can be generalized for the case with more events $\overline{X_{1}}, \overline{X_{2}}, \ldots, \overline{X_{m}}$ that depend on the state of a common node. The equation for computing the probability $P\left(\overline{X_{1}} \overline{X_{2}} \cdots \overline{X_{m}}\right)$ comprises $2^{m}$ terms. To reduce the computation time, the method we propose is limited to the pairs of events depending on the state of a common node, for which the equation $(20,24$, or 29 as is the case) comprises only four terms.

This is the approximation that may slightly affect the result given by the method we propose. Also, this work does not treat those cases when two MVI terms have two or more common nodes, considering that these cases are very rare. Nevertheless, this method provides a network reliability evaluation with a very good accuracy, as can be seen in the next section. In all the checks we have made, this method has generated exact values or slightly pessimistic results. So, the reliability value given by this method can be interpreted as a lower limit and it can be explained on the basis of (25) and Remark 1 . This aspect is very important for a reliability study.

Before ending this section, an answer to the next question is required: why did this method focus only on minimal paths and not on minimal cuts at all? Indeed, it is well known that for networks with high reliability, the approaches based on minimal cuts are generally more appropriate for an approximate evaluation. However, in our case, an approach similar to that applied to the minimal paths is no longer appropriate, because when both node and link failures are considered, one must take into account a set of cuts consisting of nodes, a set of cuts consisting of links, and another one that comprises both nodes and links. So, when the nodes are also considered, the number of minimal cuts increases very much. For this reason, the proposed method is focused only on minimal paths.

\section{Numerical Results}

To illustrate the efficiency of this approximate method, we consider the network models $N_{2}$ and $N_{3}$ presented in Figure 2. For these networks, Table 4 presents comparative results, by assuming for all the nodes a reliability value of 0.98 and for all the links a reliability value of 0.95 . Observe that the proposed method gives an accurate result for the network $N_{2}$, and it gives a slightly approximate value with five accurate decimal places for the larger network $\mathrm{N}_{3}$.

Computing time for reliability evaluation is presented in Table 5. Compared to the exact method presented in Section 5, for network model $N_{3}$, the proposed approximate method greatly reduces the computational time, from $57 \mathrm{~min} 17 \mathrm{~s}$ to $18 \mathrm{~min} 43 \mathrm{~s}$.

The values presented in Table 5 highlight the rapid growth of the computation time for the reliability evaluation with an increasing network size. The methods of network reliability evaluation based on SDP algorithms fall in the NP-hard category and, consequently, are difficult to apply for very large networks, such as social networks. In these cases, other techniques for approximate evaluation can also be applied, especially the Monte Carlo simulation (see, for example, [24-27]). Even so, the methods based on SDP algorithms are still necessary for the validation of simulation programs.

\section{Final Remarks}

In this work, the problem of two-terminal network reliability evaluation in which both link and node failures are considered is discussed. An approximate method that provides a very good accuracy is proposed. Compared to an exact method, this approximate method greatly reduces the computation time for complex networks.

The proposed solution can be applied with any SDP algorithm, but the accuracy of the reliability estimation depends on the method used for transforming the set of minimal paths into a set of disjoint products. When the number of disjoint products is lower, the reliability estimation is better. For this reason, efficient MVI algorithms as NMVI or the hybrid algorithm given by Chaturvedi and Misra [23] are recommended. Another approach based on binary decision diagrams (BDDs) is also recommended [28]. 
TABLE 4: Network reliability evaluation $\left(R_{s-t}\right)$. Comparative results.

\begin{tabular}{lccc}
\hline Network model & $\begin{array}{c}\text { Source and } \\
\text { target nodes }\end{array}$ & $\begin{array}{c}\text { Exact method } \\
\text { based on NMVI }\end{array}$ & $\begin{array}{c}\text { The proposed } \\
\text { method }\end{array}$ \\
\hline$N_{2}$ & $s=1, t=13$ & 0.982883 & 0.982883 \\
$N_{3}$ & $s=1, t=20$ & 0.959579 & 0.959575 \\
\hline
\end{tabular}

TABLE 5: Computing time for reliability evaluation.

\begin{tabular}{lcc}
\hline Network model & $\begin{array}{c}\text { Exact method } \\
\text { based on NMVI }\end{array}$ & $\begin{array}{c}\text { The proposed } \\
\text { method }\end{array}$ \\
\hline$N_{2}$ & $0.1 \mathrm{~s}$ & $0.06 \mathrm{~s}$ \\
$N_{3}$ & 57 min $17 \mathrm{~s}$ & $18 \operatorname{min~} 43 \mathrm{~s}$ \\
\hline
\end{tabular}

\section{Data Availability}

The paper presents a method for network reliability evaluation for which mathematical proofs are included. It can be applied for any network, and therefore, it does not depend on specific data.

\section{Disclosure}

The same issue of two-terminal reliability evaluation in large networks is addressed by the paper "SDP Algorithm for Network Reliability Evaluation", authors P. Caşcaval and S. A. Floria, presented at the IEEE Conference INISTA 2017 [1]. In that paper, an efficient SDP method (called NMVI) for transforming algebraically a structure function (expressed in terms of minimal paths or cuts) into a sum of disjoint products is proposed. This new method is based on an MVI technique and provides better solutions, with fewer disjoint products, compared with other well-known MVI methods. The author asserts that some general issues, such as notations, nomenclature, or other general considerations on network reliability evaluation, are similar to those outlined in [1].

\section{Conflicts of Interest}

The author declares that there is no conflict of interest regarding the publication of this paper.

\section{Acknowledgments}

The author thanks his colleague Sabina-Adriana Floria for the useful and fruitful discussions. Also, many thanks are due to Dr. Florin Leon and Dr. Marius Kloetzer for the helpful suggestions which helped improve the readability of this paper.

\section{References}

[1] P. Caşcaval and S. A. Floria, "SDP algorithm for network reliability evaluation," in 2017 IEEE International Conference on INnovations in Intelligent SysTems and Applications (INISTA), Gdynia, Poland, July 2017.
[2] K. Aggarwal, J. Gupta, and K. Misra, "A simple method for reliability evaluation of a communication system," IEEE Transactions on Communications, vol. 23, no. 5, pp. 563-566, 1975.

[3] K. K. Aggarwal, K. B. Misra, and J. S. Gupta, "A fast algorithm for reliability evaluation," IEEE Transactions on Reliability, vol. R-24, no. 1, pp. 83-85, 1975.

[4] J. A. Abraham, "An improved algorithm for network reliability," IEEE Transactions on Reliability, vol. R-28, no. 1, pp. 58-61, 1979.

[5] S. Soh and S. Rai, "CAREL: computer aided reliability evaluator for distributed computing networks," IEEE Transactions on Parallel and Distributed Systems, vol. 2, no. 2, pp. 199213, 1991.

[6] M. Veeraraghavan and K. S. Trivedi, "An improved algorithm for symbolic reliability analysis," IEEE Transactions on Reliability, vol. 40, no. 3, pp. 347-358, 1991.

[7] J. S. Provan and M. O. Ball, "Computing network reliability in time polynomial in the number of cuts," Operations Research, vol. 32, no. 3, pp. 516-526, 1984.

[8] M. O. Ball and J. S. Provan, "Disjoint products and efficient computation of reliability," Operations Research, vol. 36, no. 5, pp. 703-715, 1988.

[9] K. S. Trivedi, Probability and Statistics with Reliability, Queueing and Computer Science Applications, John Wiley \& Sons, New York, NY, USA, 2002.

[10] F. Beichelt and L. Spross, "An effective method for reliability analysis of complex systems," Journal of Information Processing and Cybernetics, vol. 23, pp. 227-235, 1987.

[11] P. Caşcaval and B. F. Romanescu, "Complementary approaches for the network reliability evaluation: network decomposition and Monte Carlo simulation," in Bul. Inst. Polit. Iaşi, Tomul L (LIV), Fasc. 1-4, pp. 123-131, Automatică şi Calculatoare, 2004.

[12] P. Caşcaval and A. R. Macovei, "Reliability evaluation by network decomposition," in Bul. Inst. Polit. Iaşi, Tomul XLIX (LIII), Fasc. 1-4, pp. 56-65, Automatică şi Calculatoare, 2003.

[13] P. Caşcaval and B. A. Botez, "Recursive algorithm for twoterminal network reliability evaluation," in Bul. Inst. Polit. Iasi, LI (LV), Fasc. 1-4, pp. 137-146, Automatică şi Calculatoare, 2005.

[14] K. B. Misra, Reliability Analysis and Prediction: A Methodological Oriented Treatment, Elsevier, Amsterdam, Oxford, New York, Tokyo, 1992.

[15] M. Shooman, Reliability of Computer Systems and Networks: Fault Tolerance, Analysis, and Design, John Wiley \& Sons, New York, NY, USA, 2002.

[16] S. K. Chaturvedi, Network Reliability: Measures and Evaluation, Scrivener Publishing-Wiley, Hoboken, NJ, USA, 2016.

[17] Y. Shen, "A new simple algorithm for enumerating all minimal paths and cuts of a graph," Microelectronics Reliability, vol. 35, no. 6, pp. 973-976, 1995.

[18] R. Mishra, M. A. Saifi, and S. K. Chaturvedi, "Enumeration of minimal cutsets for directed networks with comparative reliability study for paths or cuts," Quality and Reliability Engineering International, vol. 32, no. 2, pp. 555-565, 2016.

[19] F. Beichelt and L. Spross, "An improved Abraham-method for generating disjoint sums," IEEE Transactions on Reliability, vol. R-36, no. 1, pp. 70-74, 1987. 
[20] M. O. Locks, "A minimizing algorithm for sum of disjoint products,” IEEE Transactions on Reliability, vol. R-36, no. 4, pp. 445-453, 1987.

[21] K. D. Heidtmann, "Smaller sums of disjoint products by subproduct inversion," IEEE Transactions on Reliability, vol. 38, no. 3, pp. 305-311, 1989.

[22] T. Luo and K. S. Trivedi, "An improved algorithm for coherent-system reliability," IEEE Transactions on Reliability, vol. 47, no. 1, pp. 73-78, 1998.

[23] S. K. Chaturvedi and K. B. Misra, "A hybrid method to evaluate reliability of complex networks," International Journal of Quality \& Reliability Management, vol. 19, no. 8/9, pp. 10981112, 2002.

[24] K. F. Tee, L. R. Khan, and H. Li, "Application of subset simulation in reliability estimation of underground pipelines," Reliability Engineering \& System Safety, vol. 130, pp. 125-131, 2014.

[25] K. M. Zuev, S. Wu, and J. L. Beck, "General network reliability problem and its efficient solution by subset simulation," Probabilistic Engineering Mechanics, vol. 40, pp. 25-35, 2015.

[26] H.-S. Li, Y.-Z. Ma, and Z. Cao, "A generalized subset simulation approach for estimating small failure probabilities of multiple stochastic responses," Computers \& Structures, vol. 153, pp. 239-251, 2015.

[27] A. Birolini, Reliability Engineering, Theory and practice, Springer-Verlag, Berlin Heidelberg, 2014.

[28] X. Zang, H.-R. Sun, K. S. Trivedi, and D. R. Avresky, Eds., “A BDD approach to dependability analysis of distributed computer systems with imperfect coverage," in Dependable Network Computing, pp. 167-190, Kluwer Academic Publishers, Amsterdam, Netherlands, 1999. 


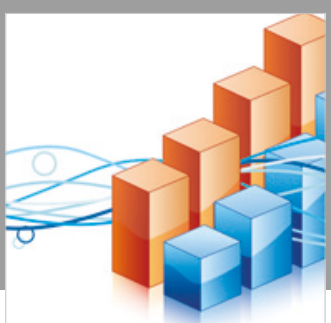

Advances in

Operations Research

\section{-n-m}
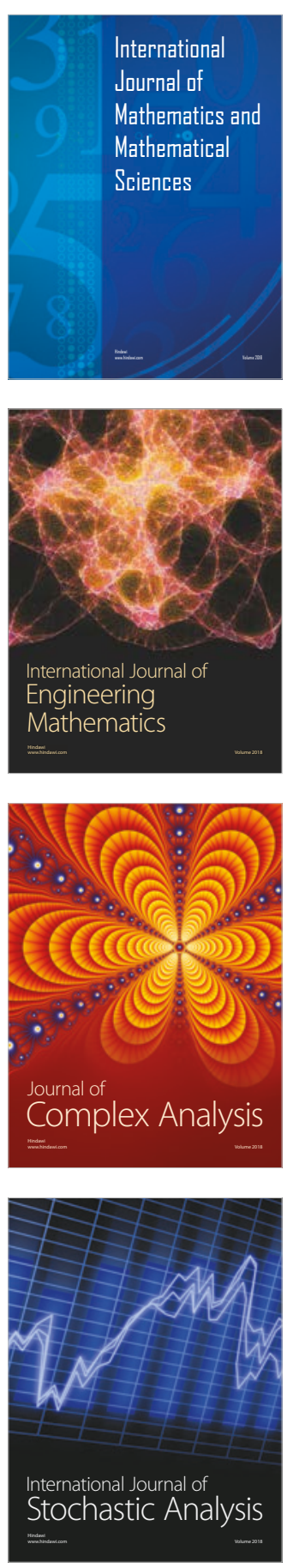
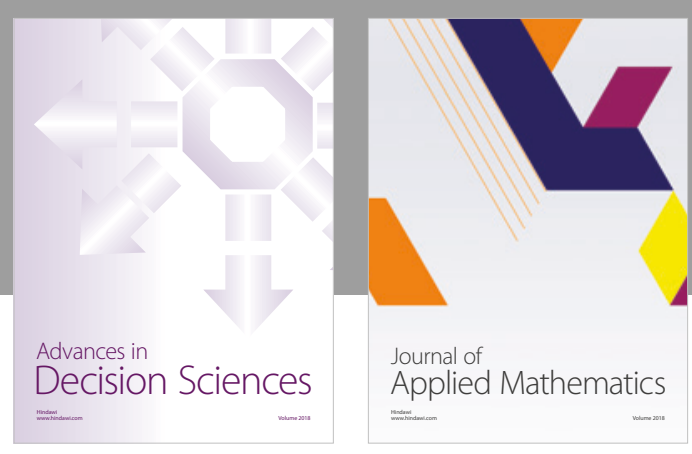

Journal of

Applied Mathematics
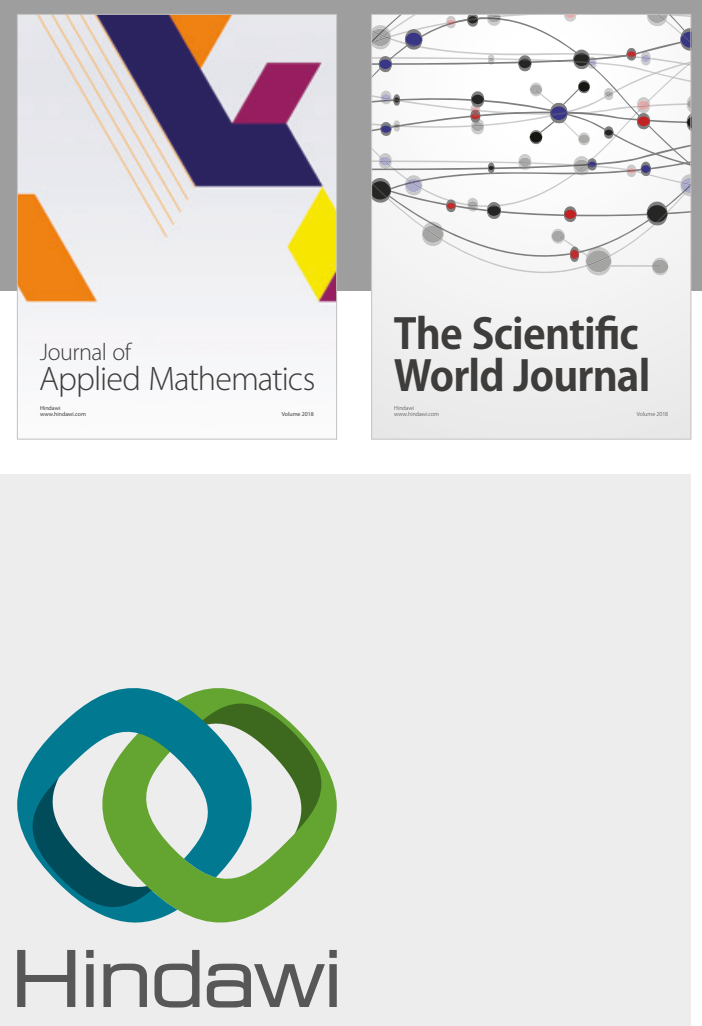

Submit your manuscripts at

www.hindawi.com

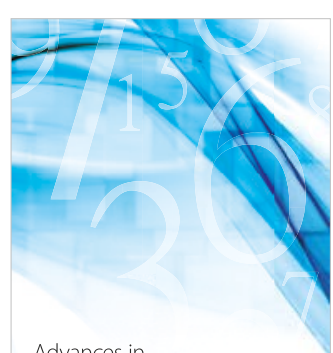

Advances in
Numerical Analysis
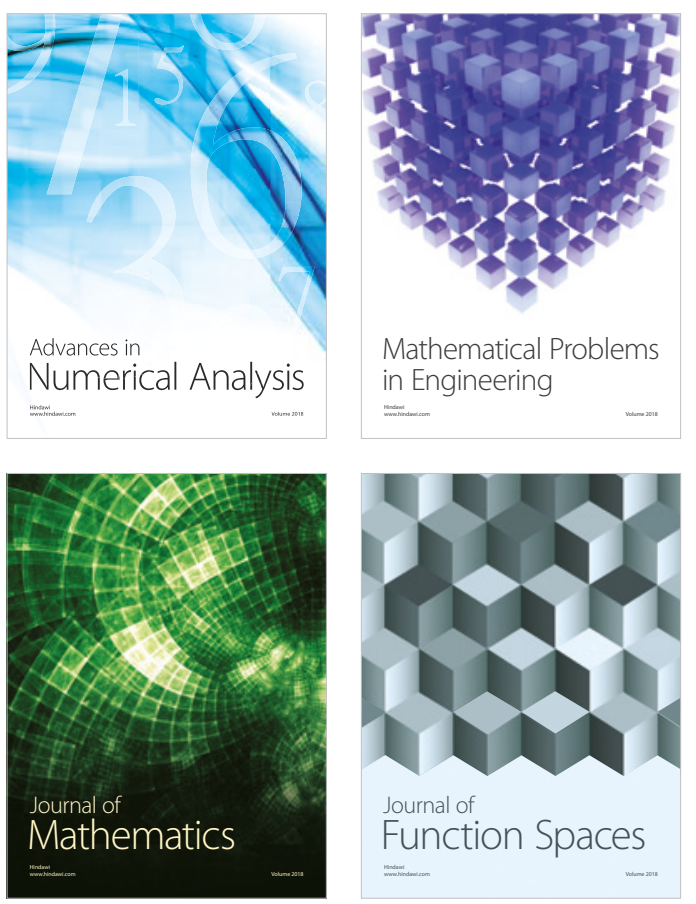

Mathematical Problems in Engineering

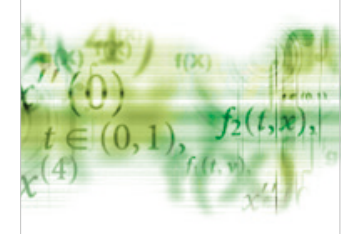

International Journal of

Differential Equations

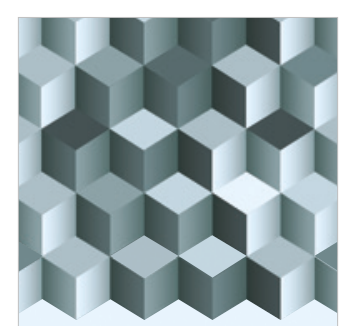

Journal of

Function Spaces
The Scientific

World Journal

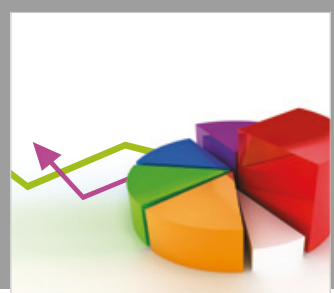

Journal of

Probability and Statistics
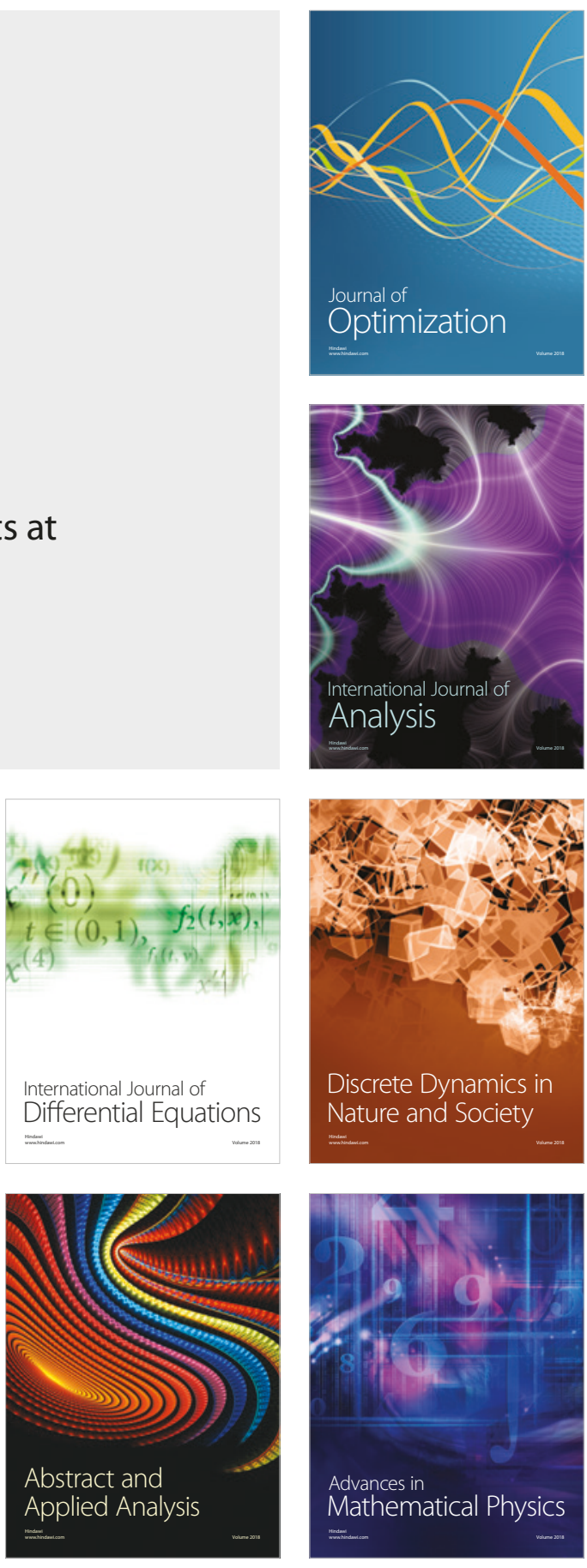